\title{
TRIC-track: Tracking by Regression with Incrementally Learned Cascades
}

\author{
Xiaomeng Wang \\ Michel Valstar \\ Brais Martinez \\ Muhammad Haris Khan \\ Tony Pridmore \\ Computer Vision Laboratory, University of Nottingham, Nottingham, UK \\ \{psxxw, michel.valstar, brais.martinez, psxmhk, tony.pridmore\}@nottingham.ac.uk
}

\begin{abstract}
This paper proposes a novel approach to part-based tracking by replacing local matching of an appearance model by direct prediction of the displacement between local image patches and part locations. We propose to use cascaded regression with incremental learning to track generic objects without any prior knowledge of an object's structure or appearance. We exploit the spatial constraints between parts by implicitly learning the shape and deformation parameters of the object in an online fashion. We integrate a multiple temporal scale motion model to initialise our cascaded regression search close to the target and to allow it to cope with occlusions. Experimental results show that our tracker ranks first on the CVPR 2013 Benchmark.
\end{abstract}

\section{Introduction}

We propose a method for structured object Tracking by Regression with Incrementally learned Cascades (TRICtrack). Visual tracking of generic objects is one of the most active topics in computer vision. It aims to detect the location of a possibly moving target by extracting local appearance features and matching them between consecutive images to obtain accurate estimates of target location, often helped by similar estimates of the object's motion. The parameters describing a target object can vary, but often include position, size, orientation and velocity. Tracking information can subsequently be used to reason about the target's behaviour or complete other tasks that require knowledge of the object's state. If solved, visual tracking has extensive applications in areas such as visual (robot) navigation, surveillance, traffic monitoring, video compression, medical imaging etc.

Despite the large body of computer vision work addressing this problem, robust visual tracking of generic objects is still a challenging problem. The performance of a visual tracking algorithm is affected by many factors, such as non-rigid object deformation, partial or full occlusion of the target, illumination variation, scale variation, viewpoint

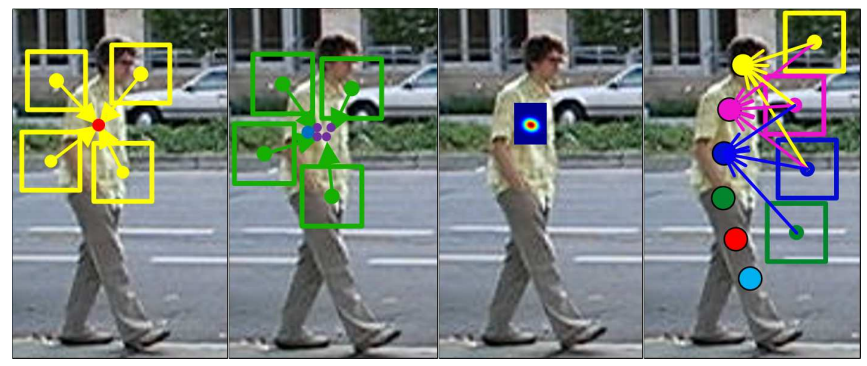

(a)

(b)

(c)

(d)

Figure 1: (a) Training a direct displacement regressor with four examples. (b) Testing a regressor. Four test patches sampled around the initial location (blue dot) provide predictions (purple dots). (c) Evidence aggregation map. (d) Location-based initialisation and implicit shape model.

change, motion blur, background clutter, etc. The combination of rigid motion and non-rigid object deformation results in complex appearance changes, making general object tracking a particularly hard problem.

Current part-based trackers rely upon a response map estimating the likelihood that any given location in an image represents the target (part). While approaches to the computation of this fitness vary from simple template matching to complex machine learning based methods, all assign a single scalar value to a queried location. Tracking then becomes a problem of determining what area(s) to search in, often guided by some motion model(s), how to construct an appearance model that can capture changes in the object's image properties, and how to deal with local optima.

While a template likelihood strategy may appear to be the logical solution to the visual tracking problem, its view of the image as a set of independent, possible target locations introduces a number of inherent drawbacks. Firstly, incorrect optima occur when changes in the target's appearance make it look less like the original template than some background image patch(es), or when there are mul- 
tiple identical objects (parts) in the scene. Secondly, for the template strategy to work, an entire region of interest needs to be searched. This can be computationally intensive, but methods (e.g. gradient descent) introduced to speed up the search can also converge to local optima. Thirdly, in a template likelihood strategy local information (be it part of the object foreground or the background) only serves to inform the tracker whether it is the target or not. Once the optimal location has been sampled, sampling additional nonoptimal local patches does not improve the confidence that this is indeed the target location. It can only make matters worse: increasing the size of the search area only increases the likelihood of falling upon an incorrect optimum. Finally, template likelihood approaches to part-based tracking cannot directly use the appearance of one part to determine the location of another. Although an explicit structure model can be learned to constrain the expected relative positions of parts, such a shape fitting step is effectively bolted-on on top of independent, individual local part trackers.

We propose to replace this local fitness-based approach by direct displacement-based tracking in which we predict the two-dimensional displacement vector between the centre of a sampled image patch and the target (part) location using regressors (see Fig. 1). In doing so, local patches contribute to the solution by directly 'voting' for the target (part) location. In addition, while template-based approaches need to model part appearance and shape fitness separately, our direct displacement prediction by regression tracker implicitly learns the shape and possible deformations of an object. It does so by tracking each part using not only the local evidence for that part, but also evidence provided by neighbouring parts.

We adopt cascaded regression [3, 22], specifically the Supervised Descent Method (SDM)[22], which has been successfully applied to the localisation of facial points, for which models were learned offline on very large sets of faces. Cascaded regression makes increasingly smaller steps towards the target location, each regressor in the cascade being trained on a smaller region around the ground truth and thus having a smaller expected error. While SDM has been used for what is essentially structured object detection, it has never been used for online model-free tracking. The key difference between detection of a known object and generic object tracking is that appearance and structure models of the former can be learned offline on potentially hundreds of thousands of images, while the models for the latter must be initialised on a single frame.

To learn the appearance changes of our parts over time, we take inspiration from the incremental learning of cascaded regression proposed by Asthana et al. [1]. That work personalises SDM for facial point localisation, initialising the SDM offline on a large database of faces, and using newly tracked faces to incrementally update it. In our ap- proach the SDM is initialised on the first frame only, and we use Local Evidence Aggregation [14] of the separate regression contributions to provide a confidence level, which is used to decide whether we can use the tracking result to update the SDM.

We report on extensive tests, both internal evaluations to determine the relative value of the various components of our proposed tracker, and comparisons against the current state-of-the-art. Traditional benchmark measures [21] assume the target is represented by a bounding box. This is not the case here. Deformable articulated objects often display significant non-rigid deformation during tracking. As a result, parts of the object would either 'stick out' of the bounding box, or the bounding box would contain large areas of background. The method presented here therefore tracks points, not boxes. This means that the best way to evaluate our system is to compare the predicted and ground truth locations of target parts. However, as this information is not available for the state-of-the-art, we revert to comparison based on a retro-fitted bounding box.

In summary, we make the following four contributions:

- We propose direct displacement prediction for the first time in online model-free tracking. By aggregating samples' predictions, we obtain a robust prediction distribution.

- We implicitly model the shape of a target using local evidence from multiple parts.

- We adapt the framework of SDM [22] to the problem of online tracking of generic objects. We show that it is possible to learn the cascade models on-the-fly without strong supervision.

- We integrate a multiple temporal scale motion model [11], taking the proposed method beyond 'tracking by detection'.

\section{Related Work}

We divide the related work in two: structured object tracking-by-detection methods, in which the similarity is the aim to track a structured object, and works in which regressors were used to perform object alignment. We finally highlight the similarities and differences of our approach with respect to these methods.

Structured tracking-by-detection: Imposing shape structure on a part-based object model is typically done by combining part-based appearance models and a global shape model. Given a set of location hypotheses for each part (e.g. particles in Particle Filtering or a Region of Interest in a sliding-window search), the appearance models give a likelihood to each hypothesis. The final hypothesis finds a compromise between maximising the individual responses and 
minimising the restrictions imposed by the shape model. Since the number of possible outputs grows exponentially with the number of parts and part hypotheses, it is most common to resort to an efficient shape model, e.g. Deformable Part Models (DPM) [7], where exact inference is possible. These models are often extended to allow for tracking-specific purposes, most commonly using temporal consistency or online/incremental adaptation of the models.

For example, [18, 23] adapt the Implicit Shape Model [13], where each part casts an independent vote on the location of the object's centre, and the object location is obtained from their combination. The star model [4], popular in object detection, has also been adapted for tracking. For example, [12] incorporates the star model into a Bayesian filtering framework and a MCMC-based search. Alternatively, [25] used a tree-based model (in fact they define a minimum spanning tree between parts), which resulted in an equally efficient minimisation but avoided the hierarchical structure of the star model.

Another aspect characterising structured object tracking is the learning strategy followed. In the tracking-bydetection framework, classifiers are trained online using the first frame only. More recent approaches often adapt successful structured learning classifiers online. For example, [24] adapt the Latent SVM proposed in [7] to the online scenario. In this way, the parts are not manually defined but rather learnt in a weakly-supervised manner. Similarly, the very successful Struck tracker [8] and SPOT tracker [25] make use of the Structured SVM framework of [19].

Regression-based model-free tracking: The realisation that discriminatively-trained regressors could be effectively used for object localisation has been around for some time. Several methods, such as [6], [16] and [20], propose to exploit discriminatively-trained regressors to propose new object states, while a classifier was used in combination to validate the predictions. [15] also used a sequence of linear regressors for facial feature tracking. Furthermore, the successful cascade of linear regressors, popularised by [22] for face alignment, can be traced back to a model-free tracking work [27], in which the authors proposed to learn a sequence of linear regressors (referred to as predictors), each of increased precision but lower robustness. It has been shown that this step is essential for producing accurate results, as a single regressor can be trained to be either robust or precise, but not both simultaneously.

Relation to our method: Some regression-based approaches have proposed methods similar to ours. For example, [20] proposes a routine similar to TRIC-track for online learning of the regressors. Furthermore, [27] proposed the use of a linear cascade of predictors instead of a single regressor. The main differences are however that 1) we do structured object tracking, and use the novel techniques developed for structured regression of [22], 2) we combine the predictions in a robust manner using evidence aggregation [14] rather than resorting to a classifier to validate the predictions, and 3) we integrate a multiple-temporal scale motion model to initialise the search in subsequent frames.

Similarly, our method differs from other works on structured tracking-by-detection in that we use the efficient and effective model of [22] instead of more cumbersome techniques such as structured SVM. Furthermore, we do not require an explicit shape model [22] but rather impose spatial consistency implicitly. It is interesting to notice that, for face alignment (at its core a structured object alignment problem), the introduction of discriminatively-trained regression has revolutionised the state-of-the-art.

\section{Regression-based Tracker}

This section describes our method of tracking by regression with incrementally learned cascades. We first explain direct displacement prediction by regression in 3.1. Our implicit structure model is illustrated in 3.2. We then introduce the adopted framework of cascaded linear regression[22] in 3.3. The cascaded incremental learning and multiple temporal motion modelling are explained in 3.4 and 3.5.

\subsection{Direct Displacement-based Prediction}

As our tracker directly predicts the locations of a number of parts of the object by modelling the displacement from a local patch to those parts (targets), we initialise the tracker by defining part locations instead of a set of bounding boxes. Thus, $N$ initial annotated points representing corresponding part locations are used to model the object's structure in the first frame of an image sequence. The locations are denoted as $L^{*}=\left[l_{1}^{*}, \ldots l_{i}^{*}, \ldots l_{N}^{*}\right]$, where $N$ is the number of parts and $l_{i}^{*}=\left(x_{i}^{*}, y_{i}^{*}\right)$ is the ground truth location of part $i$.

In the training stage, given an image $I$ and ground truth $L^{*}$, training samples are obtained by randomly sampling around $L^{*}$. Each training sample is a local image feature $\Phi\left(I, S_{\text {train }}\right)$ extracted from a square image patch centred at sample location $S_{\text {train }} . \Phi\left(I, S_{\text {train }}\right)$ and the displacement between $S_{\text {train }}$ and the target's location $L^{*}$ are then used to train the regressor $R$ which uses image information from a testing sample to predict part location.

Similarly, in the test stage, a number of test samples $S_{\text {test }}$ are selected around an initial candidate target location $L_{\text {init }}$. The locations $L_{\text {init }}$ are determined by the multiple temporal scale motion model described in section 3.5. The regressor $R$ then predicts the displacement from these samples' locations $S_{\text {test }}$ to the target using local image feature $\Phi\left(I, S_{\text {test }}\right)$. As we have no means of determining the quality of a single prediction, we combine a number of predictions to determine where the target is. The rationale behind this is that while correct predictions will aggregate around the same location, reinforcing each other, erroneous predictions will be more or less random, [14]. 


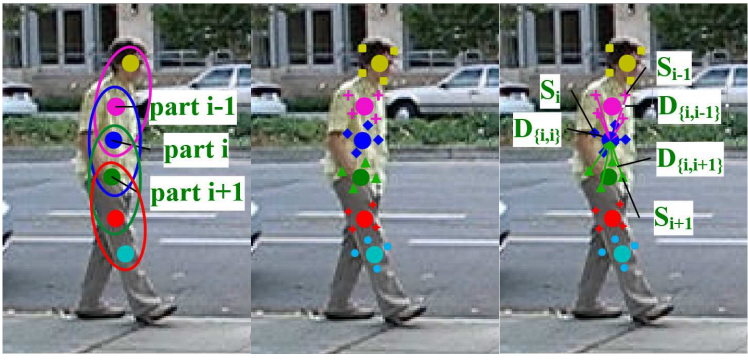

(a)

(b)

(c)

Figure 2: (a) Parts initialisation. (b) Training samples obtained around each part. (c) Implicit shape model. Three regressors estimate one part's location, each trained using features from samples around different parts.

\subsection{Implicit Shape Model}

The direct displacement prediction method provides the unique opportunity to combine local information from multiple parts, something not possible with local fitness methods. Consider part $i$ and its neighbours part $i-1$ and part $i+1$ (see Fig. 2). When seeking to locate part $i$ we train three separate regressors using image information from $S_{i-1}, S_{i}$ and $S_{i+1}$ respectively (see Fig. 2). Similarly, in the testing stage we use samples around initial candidate locations of part $i-1$, part $i$ and part $i+1$ to predict the location of part $i$. In this way, the spatial relationships between neighbouring parts are implicitly learned and applied.

Modelling groups of three parts in this way balances local and global shape. A given part's movements are closely correlated with its neighbours', while state changes in far away parts are less likely to affect the given part's. By combining our target parts in overlapping groups of three, these local shape relations effectively create a global shape model (see Fig. 2). Using image information from multiple parts also helps to avoid over-fitting. This embedding of an implicit shape model in a set of regressors is only possible because we use direct displacement prediction for tracking. Template matching approaches require the shape to be made explicit in the appearance model.

\subsection{Cascaded Linear Regression Tracker}

In our implementation of displacement prediction by regression, we adopt a cascaded linear regression framework [22], which was proposed to locate facial landmarks. Crucially, our method is not trained offline as in [22], but is initialised based on the part locations marked in frame 0 . We describe the cascaded linear regression framework used in our tracker here.

When training for a given image $I$ and initial part locations $L^{*}, M$ samples are obtained for each part by randomly sampling from a relatively close area around the part's location $l_{i}^{*}$. Sampling locations around part $i$ are denoted as $S_{i}=\left[s_{i 1}, \ldots s_{i j}, \ldots s_{i M}\right]^{T}$ where $s_{i j}$ is a 2D location in the image, $s_{i j}=\left(x_{i j}, y_{i j}\right)$. The local features $\Phi\left(I, S_{i}\right)$ are extracted from square patches centred at $S_{i}$.

We define the displacement vector (our learning goal) to be the vector from $s_{i j}$ to $l_{i}^{*}$ with $d_{i j}^{*}=\left(x_{i}^{*}-x_{i j}, y_{i}^{*}-y_{i j}\right)$. The set of all displacement vectors corresponding to $M$ samples in $S_{i}$ is $D_{i}^{*}=\left[d_{i 1}^{*}, \ldots, d_{i j}^{*}, \ldots d_{i M}^{*}\right]^{T}$. That is, $D_{i}^{*}$ is the set of displacements from $S_{i}$ to $l_{i}^{*}$. To avoid confusion, we refer to $D_{i}^{*}$ as $D_{i, i}^{*}$, so that $D_{i, i+1}^{*}$ represents the displacements from $S_{i+1}$ to $l_{i}^{*}$. This is how local shape is learned.

As we describe in section 3.2, we combine three groups of samples to predict part $i$ 's location, which means we use the features from $S_{i-1}, S_{i}$ and $S_{i+1}$ and their corresponding displacements $D_{i, i-1}^{*}, D_{i, i}^{*}$ and $D_{i, i+1}^{*}$ to train three regressors $R_{i, 1}, R_{i, 2}$ and $R_{i, 3}$ for part $i$. The trained regressors are able to map the samples' local features $\Phi\left(I, S_{i-1}\right)$, $\Phi\left(I, S_{i}\right)$ and $\Phi\left(I, S_{i+1}\right)$ to the ground truth displacement vectors $D_{i, i-1}^{*}, D_{i, i}^{*}$ and $D_{i, i+1}^{*}$, which means that the local evidence of all three sets of samples can be used to predict a single target part's location.

The regressor is obtained by minimising the difference between the predictions and the ground truth. For example, the loss functions for $R_{i, 1}, R_{i, 2}$ and $R_{i, 3}$ of part $i$ are:

$$
\begin{array}{r}
\left\|D_{i, i-1}^{*}-R_{i, 1} \Phi\left(I, S_{i-1}\right)\right\|, \\
\left\|D_{i, i}^{*}-R_{i, 2} \Phi\left(I, S_{i}\right)\right\|, \\
\left\|D_{i, i+1}^{*}-R_{i, 3} \Phi\left(I, S_{i+1}\right)\right\|,
\end{array}
$$

and the regressors are obtained by minimising these loss functions. In the following text, we refer to the group of $R_{i, 1}, R_{i, 2}$ and $R_{i, 3}$ as $R_{i}$. It is hard to find the optimal prediction of the direct displacement in one step [22]. Therefore, we learn the displacement in a cascade of steps with decreasing distance to the target. The cascaded linear regression method [22] first learns $R_{i}^{0}$, where 0 denotes the first level of the cascaded regression. Similarly, $D_{i}^{0 *}$ denotes the displacements used for training $R_{i}^{0}$. We obtain $R^{0}$ by:

$$
\begin{aligned}
& \underset{R_{i, 1}^{0}}{\arg \min }\left\|D_{i, i-1}^{0 *}-R_{i, 1}^{0} \Phi\left(I, S_{i-1}^{0}\right)\right\|+\|\omega\|^{2}, \\
& \quad \underset{R_{i, 2}^{0}}{\arg \min }\left\|D_{i, i}^{0 *}-R_{i, 2}^{0} \Phi\left(I, S_{i}^{0}\right)\right\|+\|\omega\|^{2}, \\
& \underset{R_{i, 3}^{0}}{\arg \min }\left\|D_{i, i+1}^{0 *}-R_{i, 3}^{0} \Phi\left(I, S_{i+1}^{0}\right)\right\|+\|\omega\|^{2},
\end{aligned}
$$

where $\|\omega\|^{2}$ is the regularisation term. Please note that we train each regressor with its corresponding samples and displacements. After one cascaded level, we obtain $R^{0}$ and can obtain first level predictions of part $i$ 's location by uniting 
neighbouring parts' predictions as follows:

$$
\begin{aligned}
& S_{i}^{1}=\left(S_{i-1}^{0}+R_{i, 1}^{0} \Phi\left(I, S_{i-1}^{0}\right)\right) \\
& \cup\left(S_{i}^{0}+R_{i, 2}^{0} \Phi\left(I, S_{i}^{0}\right)\right) \\
& \quad \cup\left(S_{i+1}^{0}+R_{i, 3}^{0} \Phi\left(I, S_{i+1}^{0}\right)\right)
\end{aligned}
$$

where $S_{i}^{1}$ is the first cascade level prediction of part $i$. We similarly obtain first level predictions of all other parts.

Like the first level cascaded regression, $R_{i, 1}^{1}, R_{i, 2}^{1}$ and $R_{i, 3}^{1}$ are obtained by minimising functions $4,5,6$ with $S_{i-1}^{1}$, $S_{i}^{1}$ and $S_{i+1}^{1}$ and their corresponding displacements $D_{i, i-1}^{1 *}$, $D_{i, i}^{1 *}$ and $D_{i, i+1}^{1 *}$. The $R^{2}, R^{3}, \ldots$ are learned in the same way.

In the test stage, the same number of cascaded regressors contribute to the final target location prediction $S_{\text {test } i}^{n}$, in which $n$ is the number of cascade levels. We use Local Evidence Aggregation for Regression (LEAR) [14] as a principled way of combining the individual evidences. In LEAR, each prediction contributes a unit two-dimensional Gaussian with a fixed standard deviation. LEAR then aggregates all these predictions into an un-normalised likelihood map of the target's location. The peak value of this likelihood distribution determines part $i$ 's position.

\subsection{Cascaded Incremental Learning}

One of the challenges of visual tracking is that ground truth templates, or in our case ground truth direct displacements, are available only for the first frame. Yet the appearance of tracked parts is likely to change over time, especially for deformable objects. The problem is then to decide when and how to update our appearance model (i.e. regressors) without succumbing to the model drift problem.

We use incremental learning [17] as our regressor update method. As described in 3.3, the prediction of cascaded regression results in a summation of unit Gaussians, one for every prediction made. To estimate the confidence we have in the final prediction, we divide the peak value of the likelihood map of each part by the total number of predictions. The new peak value is used to evaluate the goodness of our predictions. If it is greater than a threshold $\delta_{v}$, we say that our predictions are densely distributed. Thus, we are confident about our predictions and this tracking result can be used to update our regressors.

Turning the update of cascaded regression into cascaded incremental learning is non-trivial due to the interrelationships between the cascades: if the top-level regressor improves, this would change the training of all subsequent steps in the cascade. Asthana et al. proposed a solution to cascaded incremental learning for the problem of face alignment [1]. We follow a similar idea to learn the cascade models on the fly and combine the method with our implicit shape model to update the image information and the shape model at the same time. We however found that using a sequential incremental update provides better results than the specific incremental learning approach of [1].

We minimise Eqs. (4)-(6) to obtain $R_{i, 1}^{0}, R_{i, 2}^{0}$ and $R_{i, 3}^{0}$. For brevity we use $X_{i-1}^{0}$ to denote $\Phi\left(I, S_{i-1}^{0}\right) . R_{i, 1}^{0}, R_{i, 2}^{0}$ and $R_{i, 3}^{0}$ can be estimated by Ridge Regression [10]:

$$
\begin{aligned}
R_{i, 1}^{0}= & {\left[\left(X_{i-1}^{0}\right)^{T}\left(X_{i-1}^{0}\right)+\lambda E\right]^{-1}\left(X_{i-1}^{0}\right)^{T} D_{i, i-1}^{0 *}, } \\
& R_{i, 2}^{0}=\left[\left(X_{i}^{0}\right)^{T}\left(X_{i}^{0}\right)+\lambda E\right]^{-1}\left(X_{i}^{0}\right)^{T} D_{i, i}^{0 *}, \\
R_{i, 3}^{0}= & {\left[\left(X_{i+1}^{0}\right)^{T}\left(X_{i+1}^{0}\right)+\lambda E\right]^{-1}\left(X_{i+1}^{0}\right)^{T} D_{i, i+1}^{0 *}, }
\end{aligned}
$$

where $E$ is the identity matrix which is added to make $X^{T} X$ numerically stable.

As derived in [1], given the feature matrix $X(A)$ and displacement matrix $D(A)$, where $A$ is the number of training samples, $R(A)$ is obtained by:

$$
\begin{array}{r}
R(A)=V(A) X(A)^{T} D(A), \\
V(A)=\left[X(A)^{T} X(A)+\lambda E\right]^{-1},
\end{array}
$$

then with $B$ new training samples $X(B)$ and corresponding displacement matrix $D(B)$ obtained from the local neighbours and a given part tracked with high confidence, the updated regressor $R(A+B)$ can be found as:

$$
\begin{aligned}
& R(A+B)=R(A)-Q R(A)+ \\
& V(A+B) X(B)^{T} D(B),
\end{aligned}
$$

where,

$$
\begin{array}{r}
V(A+B)=V(A)-Q V(A), \\
Q=V(A) X(B)^{T} U X(B), \\
U=\left[E+X(B) V(A) X(B)^{T}\right]^{-1} .
\end{array}
$$

Note that the new samples added for the update of each part during incremental learning are collected around three parts at each cascade level. In this way the shape and appearance aspects of the regressors models are jointly updated, making our tracker robust to the non-rigid deformation of articulated objects.

\subsection{Multiple Temporal Scale Motion Model}

We add a multiple temporal scale motion model to our regression based tracker, which makes our method a full tracker instead of 'tracking by detection'. As we will show in the results section, displacement based regression works well when test locations are sampled relatively close to the target. Therefore, a good initial estimation of the target position is expected to significantly help to provide accurate final predictions of target position.

To counter occlusions and abrupt motion variations, Khan et al. proposed a visual tracker operating over multiple temporal scales (MTS, [11]). This framework learns 
motion models from different temporal scales of the target tracking history (i.e. already tracked frames), and applies those models at different temporal scales in the future (see Fig. 3). Here, a temporal scale is a specific sequence of moments in time e.g. $[t-4: t]$. The construction of motion models over different temporal scales provides a much richer description of the target's recent path across the image plane. When making predictions, the model set represents variations in target motion better than any single model of this set. The application of these models over multiple temporal scales in the future allows a tracker to overcome periods of occlusion of the object.

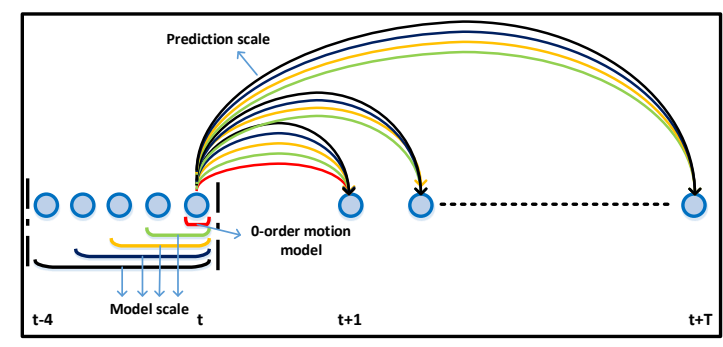

Figure 3: Multiple motion models are learned from the recent tracking history at different temporal scales, and each model is applied over multiple temporal scales in the future.

Simple motion models $\mathbf{M}$ are learned over multiple model-scales and are used to make state predictions over multiple prediction-scales, by fitting a first-order polynomial function. $\mathbf{M}$ is learned at a given model-scale separately for the $x$-location, and $y$-location of the target's state and $\mathrm{M}$ is of order 1. For instance, an $\mathbf{M}$ learned at modelscale $m$, predicts a target's $x$-location at time $t$ as:

$$
\tilde{x}_{t}=\beta_{o}^{m}+\beta_{1}^{m} t
$$

where $\beta_{1}$ is the slope, and $\beta_{o}$ the intercept. Model parameters can be learned inexpensively via weighted least squares.

A set of learnt motion models at time $t$ is denoted as $\left[\mathbf{M}_{t}^{1}, \mathbf{M}_{t}^{2}, \ldots, \mathbf{M}_{t}^{\left|\mathbf{M}_{t}\right|}\right]$, where $|$.$| is the cardinality of the set,$ which is the number of model scales. In our case, it's fixed to 4 . Each model predicts target state $l(\tilde{x}, \tilde{y})$ at $T$ prediction-scales. After applying the $\left|\mathbf{M}_{t}\right|$ models over $T$ prediction-scales, $T$ sets of motion predictions are available at time $t$.

In our part-based tracking approach, a separate MTS motion model is learned independently for each part. The $T \times\left|\mathbf{M}_{t}\right|$ motion predictions together with the prediction of the 0 -order motion model, i.e. the predicted location at time $t-1$, are available at time $t$. The top ranking motion model is used to initialise the regression search, where the ranking is determined by a classifier trained to distinguish between foreground and background patches.

\section{Evaluation}

We evaluate our proposed method of part-based tracking by regression with incrementally learned cascades (TRIC) with five experiments: four internal experiments designed to optimize the parameters for TRIC, and one experiment comparing TRIC with the state-of-the-art trackers, in which we retrofit a bounding-box around our tracked parts to be able to compare it with bounding-box ground truth.

The dataset used for evaluation of the TRIC tracker in the external experiment is the full dataset of [21]. It includes 50 video sequences and 29 state-of-the-art trackers. This benchmark provides videos with challenging conditions such as scale variation, occlusion, deformation, fast motion and so on. Thus, we can avoid over-fitting to a small subset or one specific attribute. All videos are manually tagged with what the main challenges of the video are. For example, DEF is a subset containing all videos with the attribute 'deformation - non-rigid object deformation' [21]. OCC is a subset including all videos with the attribute 'occlusion'. SV is the subset in which the videos have 'scale variation'. The internal studies are performed on 19 videos of the DEF set. We tune the parameters with the subset and then test on the full dataset to avoid over-fitting.

The performance of the various trackers is measured using precision $[2,9,21]$ and success plots. The precision plot measures 'the percentage of frames whose estimated location is within the given threshold distance of the ground truth' [21]. The success plot measures the percentage of frames for which the overlap divided by the union of the predicted and ground truth bounding boxes exceeds a given threshold ratio which varies from 0 to 1 . We report on onepass evaluation (OPE), i.e. the tracker is run throughout the whole video initialised only with the ground truth in the first frame. To rank the performance, as in [21], we use the precision obtained for a location error threshold of 20 pixels as the precision score for the precision plot. For the success plot, the area under curve (AUC) is used.

Because we predict the location of the target directly, and TRIC is a part-based tracker, for the comparison with the state-of-the-art or TRICs with different parameters, we retro-fit a bounding box. This is the same height and width as the bounding box ground truth in the first frame, and its centre location defined as the mean location of all parts.

\subsection{Parameters}

For calculation efficiency, we re-scale images using the initial frame as the reference to make every tracked target's scale approximately equal to $30 \times 30$ pixels. Specifically, the ratio is determined by $(30 * 2) /(w+h)$, where $w$ and $h$ are the bounding box groundtruth of the target. The local appearance descriptor $\Phi\left(I, S_{i}\right)$ used in our implementation is the Histogram of Oriented Gradients (HOG) [5], extracted from location $S_{i}$ with sample size $p_{s} \times p_{s}$. Because the di- 


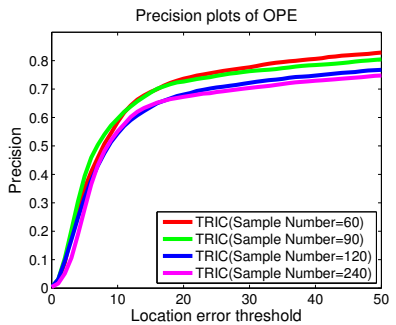

(a)

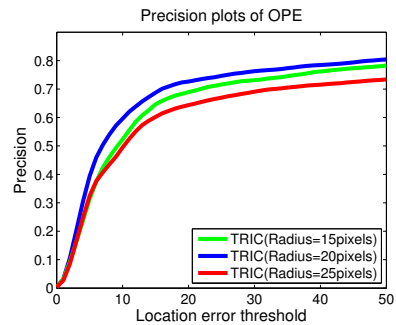

(b)

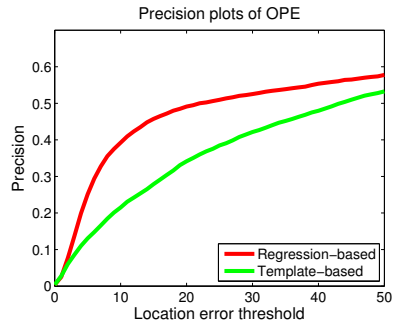

(c)

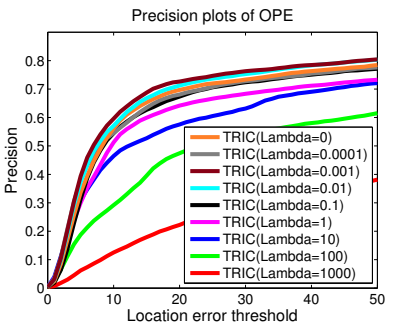

(d)

Figure 4: (a) The effect of samples number to the tracking accuracy of TRIC (sampling radius 20 pixels).(b) Effect of sampling radius of TRIC. (c) Comparison between template-based tracker and regression-based tracker. (d) Effect of regularisation parameter in ridge regression. The metric in (a),(b), (c) and (d) is the precision plot.

mensionality of the resulting feature space is relatively high, while the number of training samples is limited, we apply principal component analysis (PCA) to reduce the dimensionality of the feature. This is also helpful for avoiding over-fitting. Each sample's HOG feature is represented by $\Phi\left(I, s_{i j}, p_{s}, b\right)$ in which $b$ is the bias.

As the object is expected to move, image information acquired from the background will only give approximately correct predictions in the future, while image information derived from the object itself is expected to remain accurate over time. Thus, image patches should ideally always capture part of the foreground, which means the size of image patch is approximately equal to the implicit target part's size. The image patch size $p_{s}$ is therefore defined by: $p_{s}=\left(w_{s}+h_{s}\right) /(N-1)$, in which $w_{s}$ and $h_{s}$ are scaled $w$ and $h$. This definition ensures that the patch size is directly related to the density of target parts.

$N=6$ parts are used, as this value represents a balance between shape expressivity and computational complexity. All experiments use fixed $\delta_{v}=0.0027$ and $M=90$. The sampling radius is set to 20 pixels and the number of cascade levels is 4 . In our case, $p_{s}$ is 13 pixels. We set $\lambda=0.001$ in the Eq. in Sec. 3.4. The number of image feature dimensions kept after PCA is 30 .

\subsection{Internal Evaluation}

The internal tests are performed to optimise the internal parameters of the cascaded regressors. The first internal study examines the influence of the sampling density used in the training and test stages. The second investigates the effect of the sampling radius. The third internal study compares the performance of direct-displacement prediction by regression with a template based tracker using the same features and motion model, to investigate the cascaded regression's advantage over the template based method. The fourth internal study evaluates the effect of lambda in the ridge regression. The first, second and fourth experiments are performed with the full TRIC tracker. For the third experiment, we compare the TRIC tracker, not using the multi-temporal scale motion model and updating, with the template based tracker which uses the same features and motion model (a 0 -order motion model).

First, we investigate the effect of sampling density by setting the number of samples to $60,90,120$, and 240 with the sampling radius fixed to 20 pixels. The results are shown in Fig. 4a. The general trend clearly shows that there is not a large difference in the performance of TRIC with sample number either 60 or 90 . We choose 90 as the sample number because it is more accurate within smaller error threshold.

Second, the effect of the sampling radius is determined by varying its value in the range of 15,20 and 25 pixels (see Fig. 4b). The results clearly show that a large sampling area is more likely to include poor samples and small one is not robust enough. 20 pixels is selected to balance the accuracy and robustness of the tracker.

Third, for the comparison between the regression-based tracking method and the template-based method, we allow the latter to perform a full search in the same area from which the former samples its test locations, i.e. a circular area of radius 20 pixels. Fig. $4 c$ clearly shows the gain achieved by adopting a regression based approach.

Fourth, we exploit the effect of $\lambda$ in the Ridge Regression by setting the value of $\lambda$ to 0 and $10^{k}, k=-4: 3$. Following examination of the results shown in Fig. 4d, we chose $\lambda=0.001$ as the optimal value.

\subsection{External Evaluation}

To evaluate the TRIC tracker's performance, the TRIC tracker is tested on the whole dataset and compared with the results of all the 29 trackers included in the benchmark [21]. We also investigate the impact of different algorithm components in the TRIC tracker. TRIC-M denotes the TRIC tracker without the multiple temporal scale motion model (i.e. assuming 0-order motion) and TRIC-I tracker is the TRIC tracker without incremental learning, i.e. learning only from the first frame of the video. TRIC-S refers to 
Table 1: Performance of TRIC, without implicit shape model (TRIC-S), without incremental learning (TRIC-I), and without motion model (TRIC-M), on the DEF, OCC, SV and full dataset of the CVPR 2013 benchmark data. \# is the rank obtained against the 29 trackers in the benchmark [21], while Score is the precision score.

\begin{tabular}{ccccccccc}
\hline Trackers & \multicolumn{2}{c}{ TRIC } & \multicolumn{2}{c}{ TRIC-S } & \multicolumn{2}{c}{ TRIC-I } & \multicolumn{2}{c}{ TRIC-M } \\
\hline Dataset & $\#$ & Score & $\#$ & Score & $\#$ & Score & $\#$ & Score \\
ALL & 1 & 0.752 & 22 & 0.470 & 11 & 0.527 & 2 & 0.691 \\
DEF & 1 & 0.726 & 18 & 0.446 & 11 & 0.484 & 2 & 0.640 \\
OCC & 1 & 0.720 & 22 & 0.427 & 15 & 0.480 & 2 & 0.644 \\
SV & 1 & 0.695 & 19 & 0.464 & 13 & 0.480 & 3 & 0.648 \\
\hline
\end{tabular}

the TRIC tracker without the implicit shape model.

Results are shown in Fig. 5 for precision plot and Fig. 6 for the success plot. For clarity, only the top ten trackers are displayed. The values of the two scores for corresponding methods are included in the legends of Fig. 5 and Fig. 6.

The precision plot of TRIC tracker is about equal to the third-highest ranked tracker (SCM [26]) for a threshold up to 5 pixels, and for higher thresholds it significantly outperforms all other trackers. Struck [8] comes second. Similarly, TRIC clearly outperforms all other trackers for overlap thresholds up to 0.59 , and is approximately equal to SCM after that. One interpretation of these results is that TRIC is a robust tracker, never making very large errors, but not particularly precise. We present another interpretation, and this is that the retro-fitting of a bounding box onto the tracked parts of an articulated deformable object is unsuitable for direct comparison with bounding box trackers, as deformations of extremities (e.g. someone extending their arm) causes the mean location of the parts to move away from where the bounding box ground truth would be.

Furthermore, Table 1 shows the precision score, as well as the precision plot ranking obtained, for TRIC, TRIC-S, TRIC-I, and TRIC-M. This clearly shows the value of the implicit shape model. For DEF, without the implicit shape model, performance decreases by $28 \%$. For OCC, performance decreases by $29 \%$ without incremental learning, indicating that the incremental learning is crucial for scenarios with occlusions, and $10 \%$ without the motion model, as the motion model employed by TRIC predicts multiple frames ahead, thus overcoming brief occlusions.

\section{Conclusion}

We propose a part-based tracker employing direct displacement prediction rather than the traditional local matching of an appearance model. The method employs cascaded regression to directly predict parts' locations from local image information, learning the inference models on-the-fly. Spa-

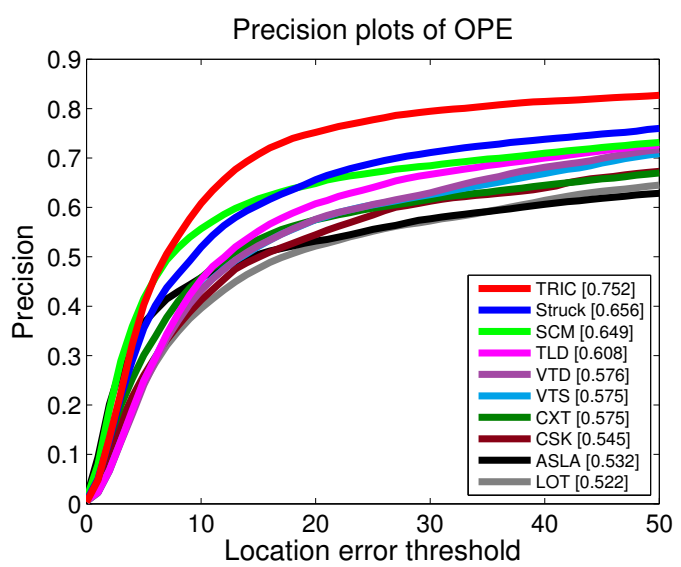

Figure 5: Precision plot of OPE for all 50 sequences.

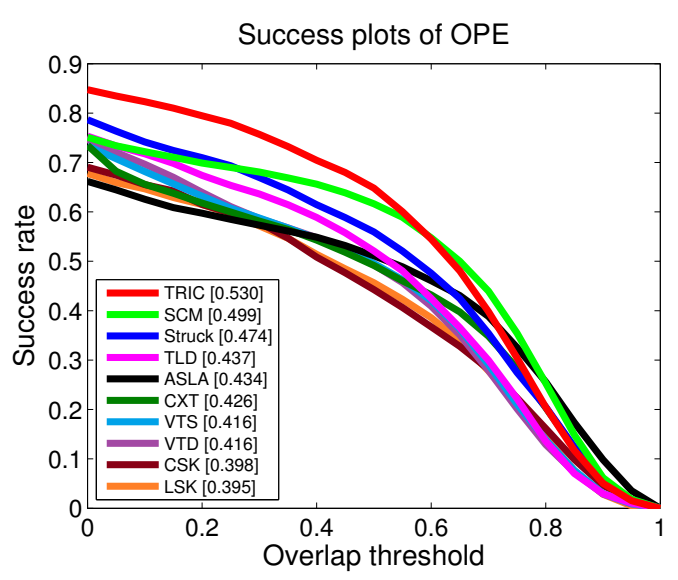

Figure 6: Success plot of OPE for all 50 sequences.

tial relationships between parts are captured implicitly by a set of regressors. We integrate a multiple temporal scale motion model to initialise our cascaded regression search close to the target and to cope with occlusions. Experimental results clearly demonstrate the value of the method's component parts, and comparison with the state-of-the-art techniques in the CVPR 2013 Visual Tracker Benchmark show that TRIC ranks first on the full dataset.

\section{Acknowledgement}

The work of Wang was supported by the China Scholarship Council and Vice-Chancellor's Scholarship for Research Excellence provided by the University of Nottingham. The work of Valstar and Martinez was supported by European Union's Horizon 2020 research and innovation programme under grant agreement No. 645378, ARIA-VALUSPA. We are grateful for access to the University of Nottingham High Performance Computing Facility. 


\section{References}

[1] A. Asthana, S. Zafeiriou, S. Cheng, and M. Pantic. Incremental face alignment in the wild. In Computer Vision and Pattern Recognition, 2014. 2, 5

[2] B. Babenko, M.-H. Yang, and S. Belongie. Robust object tracking with online multiple instance learning. Pattern Analysis and Machine Intelligence, IEEE Transactions on, 33(8):1619-1632, Aug 2011. 6

[3] T. F. Cootes, G. J. Edwards, and C. J. Taylor. Active appearance models. In Computer Vision - ECCV'98, 5th European Conference on Computer Vision, Freiburg, Germany, June 2-6, 1998, Proceedings, Volume II, pages 484-498, 1998. 2

[4] D. Crandall, P. Felzenszwalb, and D. Huttenlocher. Spatial priors for part-based recognition using statistical models. In Computer Vision and Pattern Recognition, 2005. 3

[5] N. Dalal and B. Triggs. Histograms of oriented gradients for human detection. In Computer Vision and Pattern Recognition, pages 886-893, 2005. 6

[6] L. Ellis, N. Dowson, J. Matas, and R. Bowden. Linear regression and adaptive appearance models for fast simultaneous modelling and tracking. Int'l Journal of Computer Vision, 95(2):154-179, 2011. 3

[7] P. F. Felzenszwalb, R. B. Girshick, D. McAllester, and D. Ramanan. Object detection with discriminatively trained partbased models. Trans. on Pattern Analysis and Machine Intelligence, 32(9):1627-1645, 2010. 3

[8] S. Hare, A. Saffari, and P. Torr. Struck: Structured output tracking with kernels. In Int'l Conf. Computer Vision, pages 263-270, 2011. 3, 8

[9] J. a. F. Henriques, R. Caseiro, P. Martins, and J. Batista. Exploiting the circulant structure of tracking-by-detection with kernels. In Proceedings of the 12th European Conference on Computer Vision - Volume Part IV, ECCV'12, pages 702715, Berlin, Heidelberg, 2012. Springer-Verlag. 6

[10] A. E. Hoerl and R. W. Kennard. Ridge regression: Biased estimation for nonorthogonal problems. Technometrics, 12:5567, 1970. 5

[11] M. H. Khan, M. F. Valstar, and T. P. Pridmore. Mts: A multiple temporal scale tracker handling occlusion and abrupt motion variation. In Asian Conf. on Computer Vision, 2015. 2,5

[12] J. Kwon and K. M. Lee. Highly nonrigid object tracking via patch-based dynamic appearance modeling. Trans. on Pattern Analysis and Machine Intelligence, 35(10):2427-2441, 2013. 3

[13] B. Leibe, A. Leonardis, and B. Schiele. Robust object detection with interleaved categorization and segmentation. Int'l Journal of Computer Vision, 77:259-289, 2008. 3

[14] B. Martinez, M. Valstar, X. Binefa, and M. Pantic. Local evidence aggregation for regression based facial point detection. Transactions on Pattern Analysis and Machine Intelligence, 35(5):1149-1163, 2013. 2, 3, 5

[15] E.-J. Ong and R. Bowden. Robust facial feature tracking using shape-constrained multiresolution-selected linear predictors. IEEE Trans. Pattern Anal. Mach. Intell., 33(9):18441859, 2011. 3
[16] I. Patras and E. R. Hancock. Coupled prediction classification for robust visual tracking. Trans. on Pattern Analysis and Machine Intelligence, 32(9):1553-1567, 2010. 3

[17] D. A. Ross, J. Lim, R.-S. Lin, and M.-H. Yang. Incremental learning for robust visual tracking. Int'l Journal of Computer Vision, 77(1-3):125-141, 2008. 5

[18] S. Shahed Nejhum, J. Ho, and M.-H. Yang. Visual tracking with histograms and articulating blocks. In Computer Vision and Pattern Recognition, 2008. 3

[19] I. Tsochantaridis, T. Joachims, T. Hofmann, and Y. Altun. Large margin methods for structured and interdependent output variables. Journal of Machine Learning Research, 6:1453-1484, 2005. 3

[20] O. Williams, A. Blake, and R. Cipolla. Sparse bayesian learning for efficient visual tracking. Trans. on Pattern Analysis and Machine Intelligence, 27(8):1292-1304, 2005. 3

[21] Y. Wu, J. Lim, and M.-H. Yang. Online object tracking: A benchmark. In Computer Vision and Pattern Recognition, 2013. 2, 6, 7, 8

[22] Xuehan-Xiong and F. De la Torre. Supervised descent method and its application to face alignment. In Computer Vision and Pattern Recognition, 2013. 2, 3, 4

[23] M. Yang, J. Yuan, and Y. Wu. Spatial selection for attentional visual tracking. In Computer Vision and Pattern Recognition, 2007. 3

[24] R. Yao, Q. Shi, C. Shen, Y. Zhang, and A. van den Hengel. Part-based visual tracking with online latent structural learning. In Computer Vision and Pattern Recognition (CVPR), 2013 IEEE Conference on, pages 2363-2370. IEEE, 2013. 3

[25] L. Zhang and L. van der Maaten. Structure preserving object tracking. In Computer Vision and Pattern Recognition, pages 1838-1845, 2013. 3

[26] W. Zhong. Robust object tracking via sparsity-based collaborative model. In Proceedings of the 2012 IEEE Conference on Computer Vision and Pattern Recognition (CVPR), CVPR '12, pages 1838-1845, Washington, DC, USA, 2012. IEEE Computer Society. 8

[27] K. Zimmermann, J. Matas, and T. Svoboda. Tracking by an optimal sequence of linear predictors. Trans. on Pattern Analysis and Machine Intelligence, 31(4):677-692, 2009. 3 